Relations industrielles

Industrial Relations

\title{
Atlas de l'emploi : Ville et île de Montréal, par Claude Marois, Montréal, Les Presses de l’Université du Québec, 1972, 184 pp.
}

\section{Bertrand Belzile}

Volume 28, numéro 3, 1973

URI : https://id.erudit.org/iderudit/028438ar

DOI : https://doi.org/10.7202/028438ar

Aller au sommaire du numéro

Éditeur(s)

Département des relations industrielles de l'Université Laval

ISSN

0034-379X (imprimé)

1703-8138 (numérique)

Découvrir la revue

Citer ce compte rendu

Belzile, B. (1973). Compte rendu de [Atlas de l'emploi : Ville et île de Montréal, par Claude Marois, Montréal, Les Presses de l’Université du Québec, 1972, 184 pp.] Relations industrielles / Industrial Relations, 28(3), 675-676.

https://doi.org/10.7202/028438ar

Tous droits réservés @ Département des relations industrielles de l'Université Laval, 1973
Ce document est protégé par la loi sur le droit d'auteur. L'utilisation des services d'Érudit (y compris la reproduction) est assujettie à sa politique d'utilisation que vous pouvez consulter en ligne.

https://apropos.erudit.org/fr/usagers/politique-dutilisation/ 
intellectuelle des jeunes comme objectif, le manuel, tel quel, n'a pas une très grande valeur. Il ne peut être utilisé à cette fin qu'à la condition que le cadre conceptuel de fond et le contenu soient présentés aux étudiants d'une manière critique. Si cette condition est remplie, il peut être un bon manuel.

\section{Jean-Marie RAINVILLE}

Université de Montréal

Operations Research for Managerial Decisions, by Donald R. Plane and Gary A. Kochenberger, Homewood, Ill., Richard D. Irwin Inc., 1972, $321 \mathrm{pp}$.

L'administrateur doit prendre des décisions face à des situations, à des problèmes souvent complexes par le nombre de paramètres qu'ils contiennent, de sorte qu'une solution intuitive comporte de grands risques.

La recherche opérationnelle, c'est l'application de l'analyse quantitative aux problèmes des décisions administratives. C'est la mathématique au service de la prise de décision. L'analyste ou le «spécialiste » de la recherche opérationnelle peut aider le dirigeant à systématiser l'analyse d'un problème et la prise de décision. Il vient épauler, à l'aide d'un outil de rationalisation, le dirigeant qui doit prendre une décision. C'est ce dernier cependant qui toujours prend la décision et non l'analyste qui intervient que pour mieux éclairer le dirigeant.

J'ose espérer que l'intention des auteurs n'est pas de remettre en doute la nature de cette relation lorsqu'ils écrivent qu'ils ne voient pas de distinction significative entre la science du management et la recherche opérationnelle.

Ecrit pour des non-mathématiciens qui désirent plus qu'une connaissance de surface ou des « recettes de l'analyse quantitative, cet ouvrage se veut être avant tout une discussion du sens commun et des possibilités d'utilisation des nombres en vue d'aider le dirigeant faisant face à des problèmes décisionnels.

Même s'il s'adresse à des «profanes », Operations Research for Managerial Decisions utilise quantité de formules mathématiques. Cependant, avant d'utiliser des concepts tels que le calcul différentiel et les probabilités, les auteurs prennent soin d'en présenter auparavant, les concepts de base.

Plus qu'une simple présentation théorique de la recherche opérationnelle, l'ouvrage à le souci du lecteur en ce sens qu'il cherche à développer, chez le lecteur, une certaine habileté dans la formulation des problèmes en termes de modèles mathématiques. Pour favoriser ce transfert, les auteurs mettent, tout au long du bouquin, l'emphase sur les techniques les plus utilisées dans leur application aux problèmes administratifs. Les exemples abondants et les problèmes qu'on retrouve à la fin de chacun des chapitres favorisent cet apprentissage.

$\mathrm{Au}$ plan du contenu, la programmation linéaire - terme utilisé pour décrire un grand nombre de techniques visant à optimiser ou trouver la meilleure valeur d'une fonction (objective function), en tenant compte des contraintes ou des exigences - retient l'attention principale des auteurs qui, auparavant, ont pris soin de nous instruire sur la nature de la recherche opérationnelle et d'esquisser les éléments principaux d'une théorie de la décision. Le dernier chapitre (chap. 9) du livre est consacré à la simulation comme moyen, de plus en plus intéressant à mesure que croît la complexité des systèmes, pour analyser les problèmes décisionnels.

En résumé, cet ouvrage convient à tout administrateur qui désire prendre un contact « concret» avec un outil d'analyse des problèmes impliquant une décision.

\section{Jean-Louis LANGEVIN}

\section{Université Laval}

Atlas de l'emploi : Ville et île de Montréal, par Claude Marois, Montréal, Les Presses de l'université du Québec, 1972, 184 pp.

Probablement seul un géographe est en mesure d'apprécier à sa juste valeur cet Atlas de l'emploi. Pour ma part, je trouve les 184 cartes intéressantes et non dénuées d'un certain esthétisme. II n'aurait certainement pas été inutile, cependant, que l'auteur explique davantage, ou mieux, la façon d'interpréter les ré- 
sultats. Par exemple, lorsque dix (10) municipalités contiguës possèdent le même grisé, est-ce à dire qu'elles ont toutes, chacune prise individuellement, le même nombre d'entreprises d'une certaine taille, ou plutôt que ce nombre s'applique à l'ensemble des municipalités ?

Sans doute cet atlas peut-il être utile à certains spécialistes dans des études spécifiques; je pense en particulier à des agents de développement industriel, à la recherche de sites possibles pour l'implantation de certaines entreprises. Il peut aussi fournir une bonne idée du déploiement de la main-d'oeuvre sur le territoire par secteur industriel. Je suis cependant moins confiant que l'auteur de cet instrument de travail, quant à l'utilité de celui-ci dans l'étude de la mobilité de la main-d'oeuvre. A cette fin, l'atlas devrait fournir une comparaison à deux moments du temps.

Personnellement, je souhaite, comme M. Marois lui-même, que cet atlas soit " un instrument de travail pour les chercheurs de toute discipline, qui s'intéressent à la main-d'oeuvre » (p. XIX). Je ne crois pas partager le même enthousiasme que M. Marois à cet égard.

\section{Bertrand BELZILE}

Université Laval

\section{The Politics of Comprehensive Man-} power Legislation, by Roger $\mathrm{H}$. Davidson, Policy Studies in Employment and Welfare, Number 15, Baltimore, The John Hopkins Press, 1972, 118 pp.

Depuis quelques années, les américains se préoccupent de plus en plus à décentraliser la politique de main-d'oeuvre et à en «décatégoriser》 les différents programmes. Alors que la très grande majorité des auteurs ne se contente que d'énoncer la nécessité d'une telle décatégorisation et décentralisation, très peu s'attardent à en présenter les difficultés pratiques.

Fondamentalement, cet ouvrage traite d'un des aspects de ce problème, peutêtre le plus important, à savoir l'aspect proprement politique des préoccupations en main-d'oeuvre. En effet, la mise en pratique d'une telle décatégorisation et décentralisation constitue en soi un changement tant dans la philosophie d'une politique de main-d'oeuvre que dans les structures des services aux clients. De tels changements doivent forcément être contenus dans des législations vu que les programmes à modifier sont déjà inclus dans des lois. Or c'est ici que surgit le problème politique et les conflits possibles entre technocrates spécialistes en ce domaine et les politiciens élus. Ce n'est que très rarement que ces gens sont en parfait accord sur ce qu'il y a à faire, pour des raisons évidentes. C'est pourquoi il arrive souvent qu'un projet de programme ou de loi préparé par des «spécialistes» ne soit plus reconnaissable comme tel après son adoption. Ce problème s'est présenté récemment aux Etats-Unis dans le domaine de la main-d'oeuvre. C'est ce que Davidson présente dans son livre.

Cet ouvrage caractérise en fait un nouveau courant de préoccupations en main-d'oeuvre, surtout en Amérique du Nord. En effet, la dernière décennie a été surtout marquée par l'étude des services à rendre suite à des travaux sur les imperfections du marché du travail. L'important a d'abord été de monter des programmes. Ce n'est en fait que vers la fin des années ' 60 que des préoccupations d'évaluation firent déborder les observateurs sur la nécessité de s'attarder plus longuement sur la façon dont ces services étaient rendus aux clients visés et sur leur coordination.

Fondamentalement, ce problème de coordination reste encore à résoudre et le demeurera encore un bon moment. La raison d'un tel problème semble résider dans le manque d'une acceptation universelle de la nature d'une politique de main-d'oeuvre.

L'ouvrage de Davidson a plusieurs mérites et quelques faiblesses. Débutons d'abord par les faiblesses.

L'auteur ne présente pas la conception de la politique de main-d'oeuvre selon laquelle il observe la réalité américaine et selon laquelle il fait ses suggestions. Ensuite, l'auteur s'attarde beaucoup trop longuement à faire la petite histoire du Comprehensive Manpower Act. Cela rend en fait la lecture du texte très monotone. Finalement, et cette remarque peut rejoindre notre premier point, les sugges- 